\title{
Tachistoscopic construction of four orientations of a square
}

\author{
RICHARD M. JOHNSON \\ University of Manitoba, Winnipeg, Manitoba, Canada \\ and \\ JOHN UHLARIK \\ Kansas State University, Manhattan, Kansas 66506
}

\begin{abstract}
Subjects were given repeated brief exposures to an outline of a square, a 45-deg rotation of a square (diamond), or one of two rotations of a square which were not symmetrical about the vertical axis. The stimulus forms were presented tachistoscopically for as many repetitions as were necessary for correct identification, and the nature of fragments reported prior to correct identification was examined. The obtained patterns of fragmentation were most consistent with an explanation based on the importance of the vertical axis of symmetry as opposed to explanations based on preferences for lines of certain orientations or the internal geometry of the forms.
\end{abstract}

Rotation of some two-dimensional figures in the frontal plane tends to produce distinctly different phenomenal percepts. For example, Mach (1914/1959) observed that a square and a diamond give rise to the perception of distinctly different forms, even though their internal geometry is identical. Attneave (1968) attributes this difference to competition between the axes of symmetry. In both cases, two of the axes of symmetry are bisectors oriented obliquely $45 \mathrm{deg}$ from vertical, and the other two axes of symmetry are the vertical and horizontal bisectors. Attneave suggests that the perceptual system manifests a preference for analyzing symmetry about the vertical axis and, since the square and diamond have different distributions of characteristic features about their vertical axes, different phenomenal percepts result. Rock (1973) also attributes special importance to the vertical axis of symmetry in the analysis of form. The finding that subjects judge figures to be similar on the basis of symmetry about the vertical axis, rather than about the horizontal axis, has been used to provide support for this notion (Rock \& Leaman, 1963).

A tachistoscopic repetition effect which has been recently utilized to investigate form perception (Johnson \& Uhlarik, 1974) may be useful in examining differences in the way orientation of figures affects perceived form. The repetition effect obtains when a visual stimulus is repeatedly presented for brief exposures at constant durations. Initially subjects are typically unaware of

This research was supported by grants from the Bureau of General Research, Kansas State University. The authors would like to thank Paul Goldhorn and Ross Teske for their assistance in conducting this experiment. Requests for reprints should be sent to John Uhlarik, Department of Psychology, Kansas State University, Manhattan, Kansas 66506. any aspect of the stimulus. With well-spaced repeated presentations, parts of the stimulus are reported. Gradually, these fragments become more complex. Eventually, the entire stimulus is correctly recognized on every exposure, even though the exposure duration is the same as for the initial exposures when nothing was reported. For example, the most frequent initial types of reports for presentation of a square were single vertical-line elements and parallel vertical lines. Gradually subjects reported more complex fragments, until the entire stimulus pattern was identified (Johnson \& Uhlarik, 1974). In other words, it appeared as though the subjects were gradually constructing the forms over repeated exposures.

To the extent that the fragmentation effects provide information concerning the manner in which forms are constructed from components or elements of the figure, one might expect different patterns of features to be reported as a function of the orientation. In the present study, a square, a 45-deg rotation of the square (diamond), or one of two rotations of the square which were not symmetrical about the vertical axis was repeatedly presented to subjects at initially subthreshold durations. If only the internal geometry of a form is important, one would expect no qualitative differences in the pattern of fragmentation for the four different orientations of the square. On the other hand, the distribution of features about the vertical axis of symmetry is different for the square and diamond. Therefore, the qualitative nature of the fragments reported for these two orientations of the same form should be different if the vertical axis of symmetry is the critical determinant of perceived form. The 22.5-deg rotations had no vertical axis of symmetry. In this case, one would expect fragments to be reported that are representative 
of both of the obliquely orientated axes of symmetry since these axes of symmetry are competitive in nature, according to Attneave (1968).

\section{METHOD}

\section{Subjects}

The subjects were 48 undergraduates enrolled in general psychology classes at Kansas State University. Subjects were required to have normal or corrected 20/20 vision.

\section{Stimuli and Design}

The stimulus patterns were line drawings of a square, a diamond, and a 22.5 -deg rotation (with respect to the vertical ${ }^{1}$ axis of symmetry) of the square. There were 16 subjects randomly assigned to each viewing condition. For half of the subjects in the 22.5-deg rotation condition the direction of rotation was clockwise $(+22.5 \mathrm{deg})$; for the remaining subjects rotation was counterclockwise $(-22.5 \mathrm{deg})$. Each stimulus was drawn in black ink on a white background, and subtended a visual angle of $2 \mathrm{deg}$ along its largest dimension. The width of the lines subtended 2 min of visual angle. The stimuli are shown in Figure 1.

The stimuli were individually presented in one channel of an Iconix 6131 three-channel tachistoscope. The tachistoscopic field of view was rectangular, subtending a visual angle of $5 \mathrm{deg}$ vertically and 8 deg horizontally. A homogeneous preexposure field containing a fixation point was presented in the second channel of the tachistoscope. The fixation point was centered in the preexposure field and coincided with the center of the stimulus pattern in the first channel. A homogeneous white postexposure field was presented in the third channel. The field luminance was $43 \mathrm{~mL}$ for each channel.

\section{Procedure}

The preexposure field was on at the beginning of each exposure. All subjects viewed the stimuli binocularly. The subjects were instructed to fixate on the dot in the tachistoscope and then press the button on a hand-held switch to trigger the stimulus presentation. When triggered, illumination of the preexposure field terminated and was immediately followed by illumination of the stimulus field. Immediately upon termination of the stimulus field, a postexposure field followed for $500 \mathrm{msec}$ The preexposure field then came back on until the subject initiated another exposure. The subject recorded his response and then initiated a new trial. Subjects were allowed to work

$0^{\circ}$
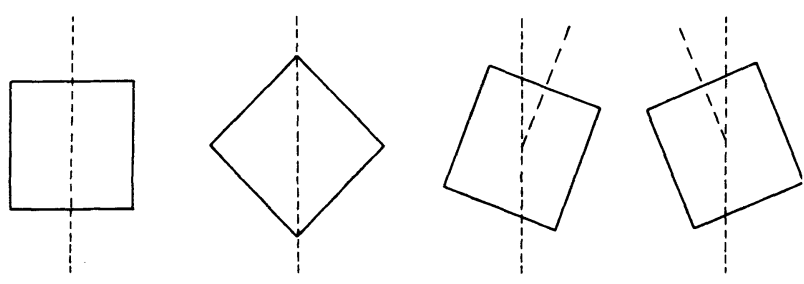

Figure 1. The four stimulus figures used in the present study. For both the square $(0 \mathrm{deg})$ and diamond $(45 \mathrm{deg})$, the dashed line represents the vertical axis of symmetry. For both of the 22.5-deg rotations, the vertical dashed line is not an axis of symmetry. However, the oblique dashed lines represent what would be the vertical axis of symmetry of the square. (These dashed lines did not appear in the stimulus presented to the subjects.) at their own pace and generally made their responses within a period of 5-10 sec. The subject was instructed to draw only what he saw. The subjects drew their responses in $2.5-\mathrm{cm}-$ square spaces, 50 of which were contained on one response sheet. Each response space subtended a visual angle of approximately $3 \mathrm{deg}$ at the subject's viewing distance. The subjects were told nothing regarding the nature of the pattern to be presented and were not told that the same stimulus would be presented on every exposure. It was emphasized that the subjects were to report only what they saw.

All stimuli were initially presented for a duration of $2 \mathrm{msec}$. This duration was increased by $1 \mathrm{msec}$ if the subject reported nothing for the first 15 exposures. If any feature was reported, the duration was held constant for 50 exposures and then increased by $1 \mathrm{msec}$. The experiment was terminated when the subject correctly reported the stimulus pattern for five exposures in succession.

\section{RESULTS}

A frequency count was made of all fragments reported for each pattern. A fragment was defined as any part of the stimulus pattern reported by the observer. Some kinds of fragments were reported with relatively low frequencies. Therefore, a criterion ${ }^{2}$ of 6 out of the 16 subjects exposed to the square and diamond, and 3 out of the 8 subjects exposed to each of the 22.5-deg rotations had to report a given fragment for it to be included in the frequency analysis. The frequencies were converted to proportions of the total number of fragments reported. These proportions are presented in Figure 2. The fragments shown along the horizontal axis of Figure 2 can be of any orientation contained in the stimulus pattern. In addition, these fragments are arranged in mean rank order of appearance. This rank order was determined by obtaining the rank order of appearance of each fragment for each subject and then taking the mean of the rank orders across all subjects.

Generally, the order of appearance of fragments progressed from simple to complex for all four patterns. An exception to this was the diamond, for which the first fragment to be reported was most often the four oblique sides without the apexes joined.

When comparing the patterns of fragmentation obtained for the square and diamond in Figure 2, three qualitative differences between the nature of fragments reported were evident. First, there were no reports of parallel lines for the diamond; second, there were no reports of three sides for the diamond; and third, there were no reports of the four lines of the square without the apexes joined. The remainder of the fragments reported for the square and diamond differed only in that their orientations were appropriate to the stimulus pattern presented. All of the types of fragments reported for the square and diamond were also reported for the 22.5-deg rotations which had no symmetry about their vertical axes.

Preferences for reporting lines of different orientations were examined for each stimulus pattern. Since all of the lines were obliquely oriented $45 \mathrm{deg}$, the 


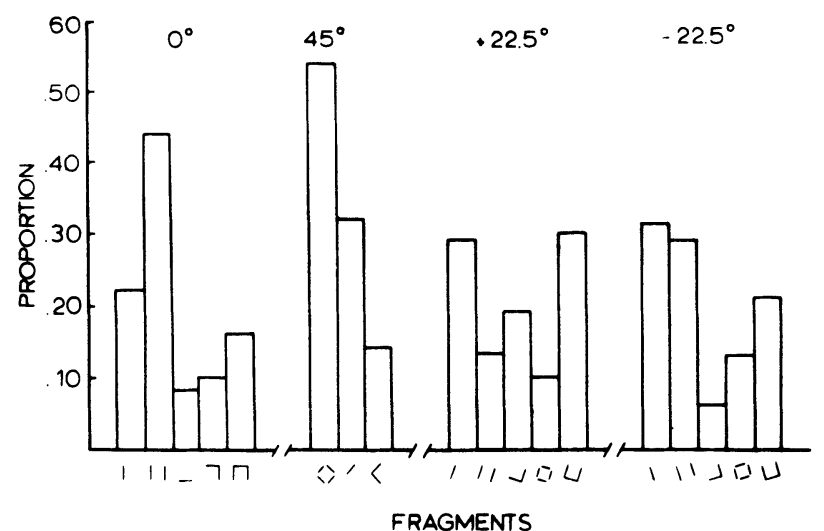

Figure 2. Proportion of different types of fragments reported for each stimulus figure. The fragments may be any orientation of that fragment contained in the stimulus. These fragments are listed in mean rank order of occurrence.

diamond was not included in this analysis. Binomial $\mathrm{z}$ tests for significance of differences between obtained and expected proportions, based on an expected value of .50 , were used for this analysis. For the square, $74 \%$ of the single lines reported were vertical, $100 \%$ of the parallel lines were vertical, and $95 \%$ of the threeline element configurations were those containing two vertical lines. Each of these obtained proportions was reliably different from what was expected due to chance $(p<.01)$. For both 22.5-deg rotations there were twoline elements which were closer to vertical and twoline elements which were closer to horizontal in orientation. For the $-22.5-\mathrm{deg}$ rotation, $100 \%$ of both the single and parallel lines, and $92 \%$ of the three-line configurations reported were those containing two lines closer to vertical. Again, each of these proportions was statistically significant $(\mathrm{p}<.01)$. For the $+22.5 \mathrm{-deg}$ rotation, $77 \%$ of the parallel lines, and $71 \%$ of the three-line configurations were closer to vertical. Both of these proportions were also statistically significant $(p<.01)$. The proportion of reports of single lines closer to vertical for the +22.5 -deg rotation was $61 \%$ and was not statistically significant. Over all three figures, $86 \%$ of the fragments reported were in the orientations that were closer to vertical.

The square reached the criterion of five successive correct reproductions at the lowest mean exposure duration $(3.2 \mathrm{msec})$. The diamond reached this criterion at a mean duration of $6.0 \mathrm{msec}$, and each of the $22.5-\mathrm{deg}$ rotations required a mean exposure duration of $6.5 \mathrm{msec}$ to reach the criterion. A one-way analysis of variance showed a significant main effect for the three patterns $[F(2,45)=23.88, p<.01]$. (The two 22.5-deg rotations were combined for this analysis.) A Duncan's range test indicated that the exposure duration at which the criterion was reached for the square was significantly different from that of the diamond and the 22.5-deg rotations $(p<.05)$. However, the exposure duration at which the criterion was reached for the diamond was not significantly different from the $22.5 \mathrm{-deg}$ rotations.
The square also reached the criterion of five successive correct reproductions in the least number of exposures $(\overline{\mathrm{X}}=67.7)$, whereas the diamond, +22.5- and -22.5-deg rotations required a mean number of exposures of $93.1,115.9$, and 115.0, respectively. An analysis of variance showed that these means were not significantly different $[F(2,45)=2.77, p>.05]$. However, the ordering of the means was consistent with the mean exposure duration at which the criterion was reached; that is, both longer durations and more exposures were required to reach the criterion for the 22.5-deg rotations.

\section{DISCUSSION}

The results of the present study are consistent with the importance that both Attneave (1968) and Rock (1973) attributed to the vertical axis of symmetry in pattern perception. The majority of fragments reported fell about the vertical axis of symmetry or the oblique axis of symmetry closest to vertical. This finding suggests that the salient dimension involved in the processing of pattern information is the vertical, rather than the horizontal axis of symmetry. Distinct qualitative differences were found between the fragments reported for the square and diamond, which would be expected, since the distribution of features about the vertical axis of symmetry is qualitatively different for a square and diamond. For example, reports of parallel lines about the vertical axis of symmetry were predominant for the square, whereas there were no reports of parallel lines for the diamond. Of course, parallel lines are not symmetrical about the vertical axis for the diamond. If the internal geometry was the major influence on the processing of form information, one would have expected the same fragments to be reported for both the diamond and the square.

The 22.5 -deg rotations were not symmetrical about a vertical axis. Each of these figures has four axes of symmetry. Two of the axes of symmetry of each figure are rotated $22.5 \mathrm{deg}$ from vertical. The other two axes of symmetry are rotated $67.5 \mathrm{deg}$ from vertical and are closer to horizontal (i.e., $22.5 \mathrm{deg}$ from horizontal). All types of fragments reported for the square and diamond were reported for both of the $22.5-\mathrm{deg}$ rotations. Since none of the axes of symmetry for either of the 22.5-deg rotations were vertical, these findings are consistent with Attneave's (1968) notion of competitive axes of symmetry. In other words, the types of fragments reported for the 22.5-deg rotations are representative of all four axes of symmetry. However, for these figures a strong tendency was found for reporting those line elements which were oriented closest to vertical. In this case symmetry refers to distribution of mirror-image fragments about a given axis. Therefore, these findings indicate a preference for reporting fragments distributed about that axis of symmetry closest to what would be the vertical axis of symmetry for a square. Thus, it appears that the axis of symmetry closest to vertical is more salient, even though fragments distributed about all axes of symmetry were reported. This preference may be a result of the square being a more familiar figure than the diamond. When asked to describe the 22.5 -deg rotations after the experiment was terminated, all subjects described them as "tilted squares" rather than "tilted diamonds." This finding supports the notion that the square may be regarded as a more familiar figure than the diamond.

An alternative interpretation of the present study could be based on preferences found for horizontally and vertically arranged stimuli in a variety of perceptual tasks. Superior performance in psychophysical acuity, discrimination, and adjustment tasks have been found in both humans and animals for stimuli aligned vertically and horizontally, as opposed to obliquely oriented stimuli (cf. Appelle, 1972). The square contained only lines of horizontal and vertical orientation, whereas 
the other three figures contained only obliquely oriented lines. Thus, the trend for the square to require lower exposure durations and fewer exposures to reach criterion is consistent with these psychophysical effects. However, one finding of the present study is inconsistent with the psychophysical findings discussed by Appelle. Stimuli of 45-deg orientations are typically found to be the least effective stimulus orientation in psycholophysical tasks. The diamond contained only lines of $45-\mathrm{deg}$ orientations. Therefore, one might expect the diamond to require the longer exposure duration and greater number of exposures to reach criterion than would be the case for any of the other figures. The results from the present study indicate that this was not the case. On the other hand, the finding that the diamond required lower exposure durations and fewer exposures to meet the criterion for correct identification, relative to the 22.5-deg rotations which had no vertical axis of symmetry, is consistent with an interpretation based on the vertical axis of symmetry. Thus, an explanation based on the variations in the axes of symmetry appears to be best able to account for the major findings of the present study.

The main findings of the present study suggest that the role of orientation in the sequential construction of fragments of a repeatedly presented visual form is consistent with previous accounts which emphasize the importance of the vertical axis of symmetry in form perception (e.g., Attneave, 1968; Rock, 1973). Since the majority of previous studies have used suprathreshold presentation of stimuli, the present study provides information which indicates that the salience of the vertical axis of symmetry is also manifested when stimuli are presented at initially subthreshold levels.

\section{REFERENCES}

Appelle, S. Perception and discrimination as a function of stimulus orientation: The "oblique effect" in man and animals. Psychological Bulletin, 1972, 78, 266-278.

Attneave, F. Triangles as ambiguous figures. American Journal of Psychology, 1968, 81, 447-453.

JoHnson, R. M., \& UHLARIK, J. J. Fragmentation and identifiability of repeatedly presented brief visual stimuli. Perception \& Psychophysics, 1974, 15, 533-538.

Масн, E. The analysis of sensations. New York: Dover, 1959. (Originally published, 1914.)

Rock, I. Orientation and form. New York: Academic Press, 1973.

Rock, I., \& Leaman, R. An experimental analysis of visual symmetry. Acta Psychologica, 1963, 21, 171-183.

\section{NOTES}

1. In the present study retinal vertical and environmental (or gravitational) vertical were the same.

2 . This criterion was based on the findings of previous research in that it represented the optimal tradeoff between the number of subjects reporting a fragment and a total number of fragments accounted for (Johnson \& Uhlarik, 1974).

(Received for publication December 9, 1976.) 